\title{
Femtosecond Pulse-Width Dependent Trapping and Directional Ejection Dynamics of Dielectric Nanoparticles
}

\author{
Wei-Yi Chiang, ${ }^{\dagger}$ Anwar Usman, ${ }^{*}{ }^{\dagger, \ddagger}$ and Hiroshi Masuhara ${ }^{* \dagger}$ \\ ${ }^{\dagger}$ Department of Applied Chemistry and Institute of Molecular Science, National Chiao Tung University, Hsinchu 30010, Taiwan \\ ${ }^{\ddagger}$ Solar and Photovoltaic Engineering Research Center, Division of Physical Sciences and Engineering, 3231-WS10 Level 3 Building 5, \\ Thuwal 23955-6900, Saudi Arabia
}

Supporting Information

ABSTRACT: We demonstrate that laser pulse duration, which determines its impulsive peak power, is an effective parameter to control the number of optically trapped dielectric nanoparticles, their ejections along the directions perpendicular to polarization vector, and their migration distances from the trapping site. This ability to controllably confine and eject the nanoparticle is explained by pulse width-dependent optical forces exerted on nanoparticles in the trapping site and ratio between the repulsive and attractive forces. We also show that the directional ejections occur only when the number of nanoparticles confined in the

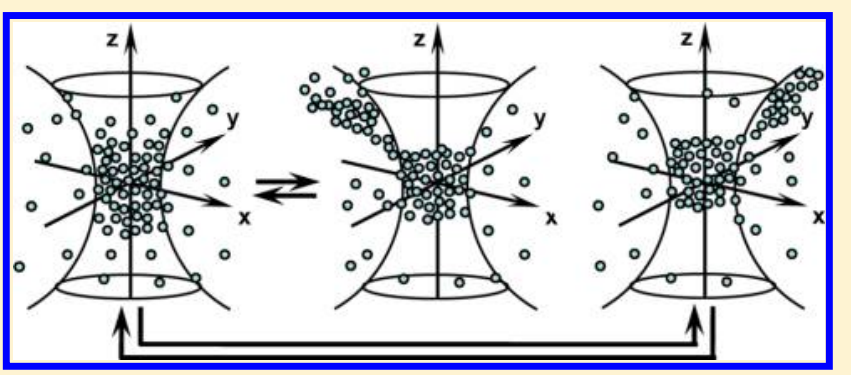
trapping site exceeds a definite threshold. We interpret our data by considering the formation of transient assembly of the optically confined nanoparticles, partial ejection of the assembly, and subsequent filling of the trapping site. The understanding of optical trapping and directional ejections by ultrashort laser pulses paves the way to optically controlled manipulation and sorting of nanoparticles.

\section{INTRODUCTION}

The dream of manipulating particles in micrometer or nanometer scales in colloidal solutions has come true with the birth of optical trapping which employs a highly focused $\mathrm{cw}$ laser beam to induce constant $\mathrm{pN}$-scale gradient force sufficient to trap the particles in three dimensions. ${ }^{1,2}$ This technique has, therefore, been applied in many research fields, and it has been showing tremendous, consistent, and increasing impacts in physics, chemistry, biology, and material sciences. ${ }^{3-8}$ Although there have been extensive applications, such as in particle depositions, ${ }^{9-12}$ particle aggregations, ${ }^{6,13-15}$ polymerizations, ${ }^{16}$ and crystallizations, ${ }^{17-19}$ it has always been a challenge for researchers to trap and manipulate single particles in nanometer-scales or even single macromolecules. ${ }^{20}$ One promising method is to induce a higher intensity of electric field at the diffraction-limited size of the focal spot, and it can be achieved by substituting $\mathrm{cw}$ laser beam with ultrashort laser pulses that possess impulsive peak powers.

The laser pulses have actually been applied in laser-induced crystallization of urea, ${ }^{21}$ glycine, ${ }^{22,23}$ and lysozyme, ${ }^{24}$ revealing that the laser pulses can also be used as a tool for controlling nucleation and growth of the amino acid or protein crystals. Optical trapping of single silica spheres with $1.28 \mu \mathrm{m}$ in diameter also indicated that the ultrashort laser pulses have an ability to trap the microscopic particle equally to that of $\mathrm{cw}$ laser. $^{25}$ Notably, 3.3-nm-sized quantum dots were stably trapped by picosecond (ps) laser pulses at the laser power as low as $100 \mathrm{~mW}$, but such optical trap could not be achieved by $\mathrm{cw}$ lasers with such level of power. ${ }^{26}$ Those results provided a hint that the advantages of optical trapping with laser pulses over the $\mathrm{cw}$ lasers can be explored when the target particles are in the Rayleigh regime. ${ }^{26}$ With this viewpoint, we have recently shown larger number or higher optical trapping ability of 50$\mathrm{nm}$-sized polystyrene or silica spheres upon replacing $\mathrm{cw}$ - with femtosecond ( $\mathrm{fs}$ ) pulsed-mode laser as the trapping beam. ${ }^{27,28}$ Furthermore, optical trapping with fs lasers have shown some astonishing features, including the split of trapping site of 60$\mathrm{nm}$-sized gold nanoparticles into two off-axis positions due to nonlinear optical effects ${ }^{29}$ and directional ejections of optically trapped 50-nm-sized polystyrene spheres out of the trapping site perpendicular to the polarization vector of the incident laser beam. ${ }^{27,30}$

The directional ejections that can be controlled by the laser pulses have been beyond the general consensus that nanoparticles are pushed in the direction of the propagating laser light. ${ }^{31,32}$ Such ejections may also be important for manipulating the nanoparticles but are yet to be developed in a practical application. In our earlier reports, theoretically, we have shown that directional ejections of the nanoparticles can occur as a result of dominating repulsive-over attractive-forces in the directions perpendicular to the polarization vector. ${ }^{27,30}$ The reasonable questions arise; e.g., how are the nanoparticles ejected from the trapping site and what parameters can control such directional ejections? On the basis of electromagnetic

Received: May 3, 2013

Revised: $\quad$ August 3, 2013

Published: August 16, 2013 
formulations, a multitude of parameters, such as the repetition rate and pulse duration of laser pulses, as well as numerical aperture of objective lens can greatly affect the repulsive and attractive forces. ${ }^{27,30}$ In addition, we also consider that particle density, particle size, particle assembly, and viscosity of the colloidal solution are other prominent factors governing the optical trapping and ejection dynamics, including the kinetic motion, gathering, ejection, and directional migration of the polystyrene nanoparticles. ${ }^{27,30}$ With the goal of elucidating the detailed behavior of the repulsive forces, in this study we examined experimentally the effects of important parameters on the directional ejections; i.e., the pulse width of fs laser pulses and particle density of the colloidal solution. We show how those two parameters control the trapping of the nanoparticles and govern their ejections from the trapping site. In general, this finding thus provides information on a technique to control the gathering and directional migration of nanoparticles by focused laser pulses, and it may open the way to optically controlled manipulation and sorting of nanoparticles from their mixtures in polydisperse colloids.

\section{EXPERIMENTAL SECTION}

2.1. Experimental Design. In these experiments, we employed an optical trapping system combined with dark-field microscopy. ${ }^{27}$ In brief, a linearly polarized fundamental mode from a Ti:sapphire laser $(800 \mathrm{~nm} ; 80 \mathrm{MHz})$, acting as a trapping beam, was passed through a prism compressor, and it was then focused by an objective lens (60×; NA 0.90) into a sample cell mounted on the stage of an inverted microscope. At the sample, the laser beam at the average power of $350 \mathrm{~mW}$ (equivalent to a light energy of $4.38 \mathrm{~nJ}$ per pulse), was concentrated on the diffraction-limited size of focal spot with the beam waist being approximately $0.46 \mu \mathrm{m}$. This means that the laser intensity at the focal spot is $527 \mathrm{GW} / \mathrm{cm}^{2}$ or equivalent to a peak intensity of $6.59 \mathrm{~kJ} / \mathrm{cm}^{2}$ per pulse.

2.2. Dark-field Microscopy. The dark-field microscopy employs white-light illumination from halogen lamp $(\lambda=400-$ $750 \mathrm{~nm}$ ) through a dark-field condenser lens (NA 1.2-1.4). With this optical setup, the same objective allows the imaging of the trapped and ejected nanoparticles onto a regular chargecoupled device (CCD) camera (JAI; CV-A55IR E) running at 30 interlaced frames per second. In order to clarify the ejected nanoparticles, the regular CCD camera was replaced by a highsensitivity CCD camera (Flovel ADT-33C), which apparently has the same detection area, but a smaller detection volume. In all measurements, before the CCD camera, the elastic light scattering originated from the laser trapping beam was completely cut by a shortwave-pass filter with transmission at $\lambda=380-720 \mathrm{~nm}$ (Semrock; Brightline 750/SP).

2.3. Sample Cell. The sample cell consisted of a silicon chamber (1 mm thickness) sandwiched between two coverglass plates (Matsunami). The inner well of the chamber (10 $\mathrm{mm}$ in diameter) was filled with a colloidal solution of polystyrene beads (PolyScience; diameter $=50 \mathrm{~nm}$, refractive index $=1.59$ at $800 \mathrm{~nm})^{33}$ suspended in distilled water (refractive index $=1.33$ at room temperature).

2.4. Pulse Width and Particle Density Parameters. The pulse width, which influences peak power of the laser pulses, was selected from 85 to $125 \mathrm{fs}$ at fwhm by tuning the optical cavity of the laser source and/or by optimizing the optical path passing through the prism compressor. In these experiments, the particle density in the colloidal solution was set at $3.8 \times$ $10^{14}$ particles $/ \mathrm{mL}$. The laser power at the sample was adjusted at the same level (350 $\mathrm{mW}$ or $4.3 \mathrm{~nJ} /$ pulse) to ensure equivalent light energy per pulse.

However, in experiments with different particle densities, the pulse width was fixed at 90 fs and the particle density was varied in the range of $(1.1-15.2) \times 10^{14}$ particles $/ \mathrm{mL}$ or $(1.1-15.2)$ $\times 10^{2}$ particles $/ \mu \mathrm{m}^{3}$. For this range, the mean distance between the centers of nanoparticles in the colloidal solutions was within the range of 250 to $100 \mathrm{~nm}$, respectively.

2.5. Data Analysis. With such an optical setup and detection system mentioned in Sections 2.1 and 2.2, only the probe light from halogen lamp scattered by the nanoparticles was detected. The positions of the nanoparticles were associated with the image of the Rayleigh scattering light detected by the CCD camera. Thus, the numbers of nanoparticles optically trapped at the trapping site and ejected to the surrounding area were assumed to be proportional to the scattering light intensity at and around the focal spot, respectively. Effects of pulse width and particle density on the optical trapping and directional ejection dynamics of the nanoparticles were analyzed from successive images from videos which were recorded for $\sim 120 \mathrm{~s}$. For the sake of repeatability, each experiment was carried out in three different noise conditions, and all data were included in analyses. The sample was then replaced by a neat solvent for baseline corrections.

\section{RESULTS}

3.1. Pulse Width Dependence. In Figure 1, panels A and $\mathrm{B}$, we show two video images of optical trapping of the

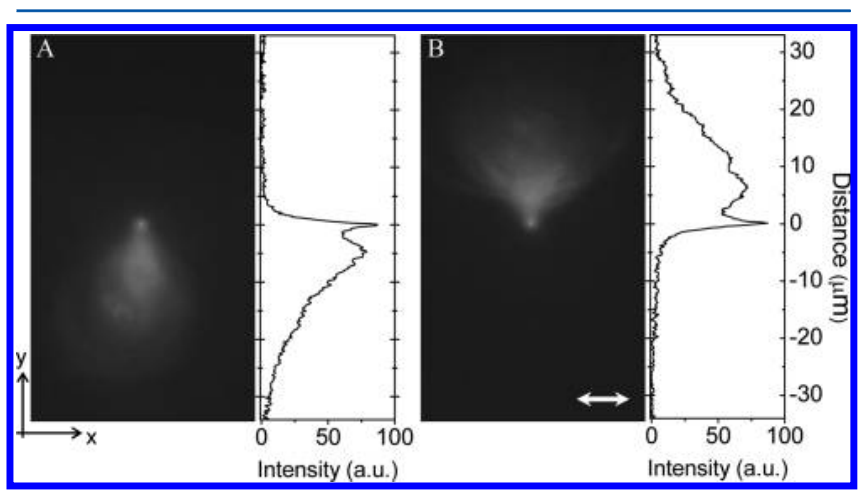

Figure 1. Two image frames (A and B) detected by a regular CCD camera and line profiles perpendicular to the polarization vector (indicated by an arrow along the $x$-axis) revealing optical trapping and ejections of polystyrene nanoparticles alternated between two directions perpendicular to the polarization vector. On the focal plane, the Poynting vector of the beam propagating along the $z$-axis decomposes into three orthogonal components $\left(S_{z}: S_{y}: S_{x}\right)$ is 1:0.05:0.0025 for the objective NA 0.9. ${ }^{30,35}$ Pulse width is $85 \mathrm{fs}$ fwhm.

nanoparticles with 85 -fs laser pulses detected by the regular CCD camera, indicating a bright spot at the beam center and bright areas perpendicular to the polarization vector. The profiles perpendicular to the polarization vector provide the scattered light intensities of the bright spots and bright areas, which are attributed to the optically trapped and ejected nanoparticles out from the trapping site along two alternated directions perpendicular to the polarization vector, respectively. $^{27}$

Figure 2 shows two halves of video images for different pulse widths from 85 to 125 fs detected by using the high-sensitivity $\mathrm{CCD}$ camera. As the detection volume is reduced, the bright 


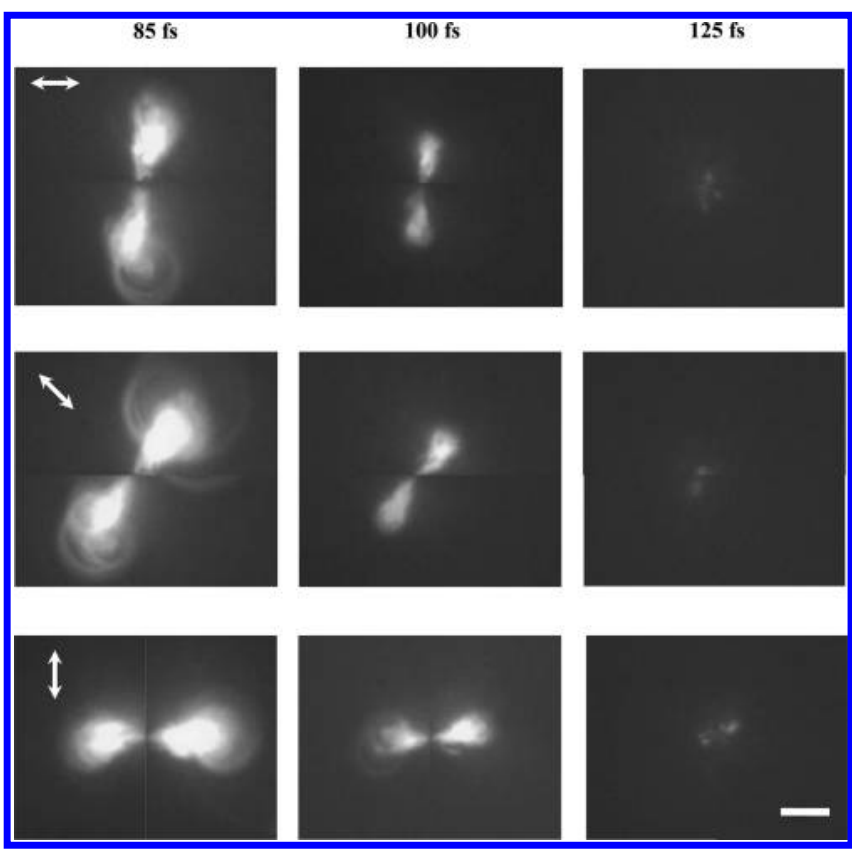

Figure 2. Combined two halves of two different CCD image frames detected by a high-sensitivity CCD camera indicating the different directional migration distances of ejected polystyrene nanoparticles out of the trapping site depending on the pulse width. The polarization vector is indicated by an arrow in each row, and the pulse width of 85 , 100 , and $125 \mathrm{fs}$ is indicated on the top of each column. The scale bar of $10 \mu \mathrm{m}$ is applied for all images.

spot is covered by the bright areas of scattered light from the ejected nanoparticles. With this CCD camera, however, the distance of their directional migrations out of the focal spot can be more precisely observed. The intensity and size of the bright areas related to number and length of the nanoparticles migrating outside the trapping site are abruptly reduced when the pulse width is widened. Typically, the maximum intensity of the bright areas decreases from 138 to 95 and 37 au, while their length decreases from 25 to 15 and $8 \mu \mathrm{m}$ from the focus point, when the pulse width is expanded from 85 to 100 and 125 fs, respectively. This indicates that the number of nanoparticles ejected out of the trapping site becomes smaller and that their directional migration distances outside the focal spot area is shrunk upon widening the pulse width. These findings, in turn, indicate that the optical trapping and ejection dynamics are definitely related to the impulsive peak power, not to the average laser power.

3.2. Particle Density Dependence. As the directional ejections of the nanoparticles are also considered as the material-side phenomenon, the particle density in the colloidal solution indeed governs the optical trapping and ejection dynamics, as shown in Figure 3 for the trapping-time dependence of the number of optically trapped nanoparticles and the directional ejections. The upper panel A shows that, at the particle density as high as $15.2 \times 10^{2} / \mu \mathrm{m}^{3}$, the trapping site is getting filled rapidly by large numbers of nanoparticles within few tens of $\mathrm{ms}$ and it is constantly filled up with the high number of nanoparticles. At this circumstance, the nanoparticles are also continuously ejected out of the trapping site along the two alternated directions perpendicular to the polarization vector (lower panel A). The probability and total duration of the two directional ejections almost balance each other for a long observation window $(\sim 120 \mathrm{~s})$. Those optical

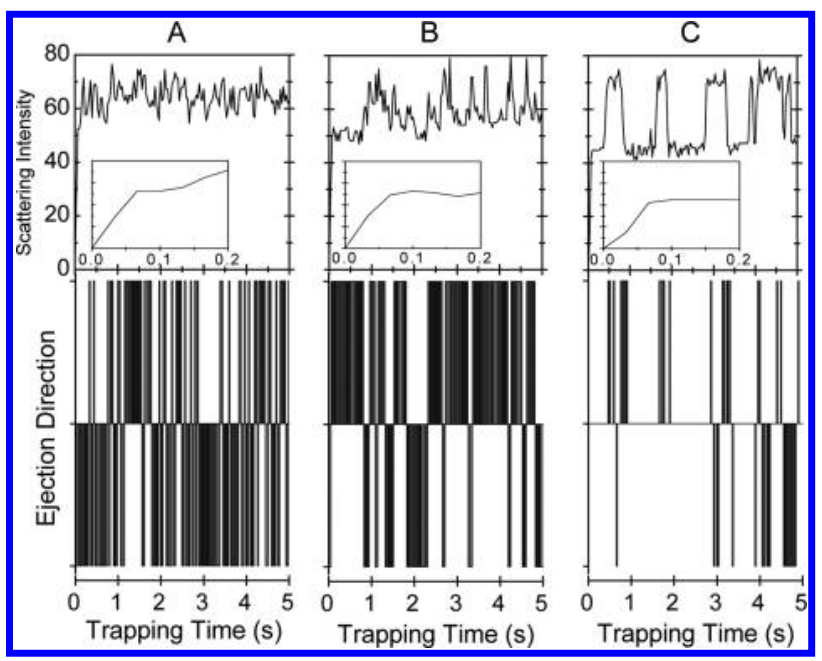

Figure 3. Upper panels: Scattering light intensity at the focal spot at different particle densities in the colloidal solution (Insets: scattering light intensity at early $200 \mathrm{~ms}$ of trapping time). Lower panels: the detected directional ejection of the nanoparticles out of the trapping site in the directions perpendicular to laser polarization. The particle density of nanoparticles is $15.2 \times 10^{2} / \mu \mathrm{m}^{3}$ for $(\mathrm{A}), 3.8 \times 10^{2} / \mu \mathrm{m}^{3}$ for (B), and $1.1 \times 10^{2} / \mu \mathrm{m}^{3}$ for (C).

trapping and ejection behaviors are almost unchanged when the particle density is decreased to $3.8 \times 10^{2} / \mu \mathrm{m}^{3}$, as shown in panel B. Upon the dilution, however, it is notable that the number of nanoparticles confined at the trapping site tends to be lower, although the trapping site is also filled rapidly by large number of nanoparticles within few tens of ms. The low particle density also leads the number of nanoparticles at the trapping site to be highly fluctuated. Such fluctuation is further evidenced in panel $\mathrm{C}$ for particle density as low as $1.1 \times$ $10^{2} / \mu \mathrm{m}^{3}$, in which the number of nanoparticles at the trapping site stochastically appears at a high level before it turns abruptly to a low level and it repeats in the same manner on subsecond scales throughout the laser irradiation. Thus, the number of trapped nanoparticles is likely fluctuated between the two levels, resembling impulse diagram as a function of time. Importantly, the directional ejections of nanoparticles out of the trapping site take place only when the number of confined nanoparticles is at the high level, or otherwise they are not observed, as shown in panel C. This indicates that the directional ejections occurred only when the number of confined nanoparticles at the trapping site is above a certain level. Original videos for data given in panels $\mathrm{A}, \mathrm{B}$, and $\mathrm{C}$ are presented in V1, 2, and 3 of the Supporting Information.

\section{DISCUSSION}

4.1. The Competition between Repulsive and Attractive Optical Forces. To evaluate pulse width dependence of the optical forces, we recall their electromagnetic formulations, which were derived from Lorentz force formalism combined with Mie scattering theory. ${ }^{27,30}$ For the incident laser pulse linearly polarized along the $x$-axis, propagating along the $z$-axis, and tightly focused by a high NA objective lens, the electric field at the focal spot is distributed into transverse and longitudinal components, which can be derived using paraxial approximation. $^{34,35}$ With the three orthogonal vectors of electric field at the focal spot, all gradient, scattering, and temporal pulse radiation forces (denoted as $F_{\text {grad }}, F_{\text {scatt }} F_{\text {temp, }}$, respectively) are acting in three-dimensions with components 
along the $\mathrm{x}^{-}, \mathrm{y}^{-}$, and $z$-axis. Those optical forces as a function of spatial positions in the focal spot, optical properties of the nanoparticles, and the parameters of laser pulse are given by the following: ${ }^{27,30}$

$$
\begin{aligned}
& \vec{F}_{\text {grad }}=-\left\{\left[\hat{x} \frac{\xi}{\left(1+4 \zeta^{2}\right)^{2}}+\hat{y}\left(\frac{K^{4} \xi^{2} \eta^{3}}{\left(1+4 \zeta^{2}\right)^{7}}\right.\right.\right. \\
& \left.-\frac{K^{4} \xi^{2} \eta}{2\left(1+4 \zeta^{2}\right)^{6}}\right) \times\left(\left(1-12 \zeta^{2}\right)^{2}+\zeta^{2}\left(6-8 \zeta^{2}\right)^{2}\right) \\
& \left.\left.+\hat{z}\left(\frac{4 K^{2} \xi^{2} \zeta}{\left(1+4 \zeta^{2}\right)^{3}}-\frac{4 K^{2} \xi^{2} \zeta\left(\xi^{2}+\eta^{2}\right)}{\left(1+4 \zeta^{2}\right)^{4}}\right)\right]\right\} \times \frac{n_{\mathrm{m}}^{2} \varepsilon_{0} \alpha E_{0}^{2}}{w_{0}} \\
& \times e^{-2\left(\xi^{2}+\eta^{2}\right) /\left(1+4 \zeta^{2}\right)} \times e^{-2 t^{2} / \tau^{2}} \\
& \vec{F}_{\text {scat }}=\left\{\hat{x} \frac{K^{2} \xi^{2}}{\left(1+4 \zeta^{2}\right)^{2}}\right. \\
& +\hat{y} \frac{K^{4} \xi^{2} \eta^{2}\left(\left(1-12 \zeta^{2}\right)^{2}+\zeta^{2}\left(6-8 \zeta^{2}\right)^{2}\right)}{\left(1+4 \zeta^{2}\right)^{6}} \\
& \left.+\hat{z} \frac{1}{1+4 \zeta^{2}}\right\} \times \frac{n_{\mathrm{m}}^{2} \varepsilon_{0} \sigma_{\mathrm{p}} E_{0}^{2}}{2} \times e^{-2\left(\xi^{2}+\eta^{2}\right) /\left(1+4 \zeta^{2}\right)} \\
& \times e^{-2 t^{2} / \tau^{2}} \\
& \vec{F}_{\text {temp }}=\left\{\hat{x} \frac{K^{2} \xi^{2}}{\left(1+4 \zeta^{2}\right)^{2}}\right. \\
& +\hat{y} \frac{K^{4} \xi^{2} \eta^{2}\left(\left(1-12 \zeta^{2}\right)^{2}+\zeta^{2}\left(6-8 \zeta^{2}\right)^{2}\right)}{\left(1+4 \zeta^{2}\right)^{6}} \\
& \left.+\hat{z} \frac{1}{1+4 \zeta^{2}}\right\} \times \frac{-2 n_{\mathrm{m}}^{3} \varepsilon_{0} \alpha E_{0}^{2} t}{c \tau^{2}} \times e^{-2\left(\xi^{2}+\eta^{2}\right) /\left(1+4 \zeta^{2}\right)} \\
& \times e^{-2 t^{2} / \tau^{2}}
\end{aligned}
$$

where $K$ is defined as $K=2 / k w_{0}$. Here, $k=2 \pi / \lambda$ is wavenumber in the medium, $n_{\mathrm{m}}$ is refractive index of medium, $\xi=x / w_{0}, \eta=$ $y / w_{0}$, and $\zeta=z / k w_{0}^{2}$ are the normalized spatial coordinates in the focal spot, $w_{0}$ is beam waist, and $\omega_{0}$ is carrier frequency of the focused beam, $\sigma_{p}=k^{2} \alpha^{4} / 6 \pi$ is scattering cross section, $\alpha=$ $4 \pi a^{3}\left[\left(\left(n_{p} / n_{m}\right)^{2}-1\right) /\left(\left(n_{p} / n_{m}\right)^{2}+2\right)\right]$ is linear polarizability, $a$ is radius, and $n_{\mathrm{p}}$ is refractive index of the nanoparticles. The impulsive peak power which is related to electric field at the central intensity peak, $E_{0}$, depends on incident laser power per pulse, $p$, and pulse width, $\tau$, as expressed by the following:

$$
E_{0}^{2}=1.7 p / \pi^{2} w_{0}^{2} n_{\mathrm{m}} \varepsilon_{0} c \tau
$$

Detailed analysis of the optical forces with numerical approaches has been discussed; by which we showed that attractive force components spatially resolved in the directions orthogonal to the polarization vector are completely overcome by respective repulsive forces. ${ }^{30}$ Thus, nanoparticles optically trapped at the focal spot can be uniformly kicked out by the repulsive forces along the directions perpendicular to the polarization vector of incident laser beam. ${ }^{27,30}$ With intense fs laser beam at the focal spot we may consider further that nonlinear optical effects such as third-order susceptibility or electro-optic Kerr effects also occur, giving nonlinear terms of polarizability in eq $1 \mathrm{a}-1 \mathrm{c}$. The third-order susceptibility effect has been observed and documented for gold nanoparticles, resulting in two off-axis trapping sites separated a few hundreds of $\mathrm{nm}$ from each other along the polarization vector. ${ }^{29}$ Thus, with the similar level of laser power of the fs pulses, the split of the trapping sites of the polystyrene beads may take place. However, since a third-order nonlinear susceptibility of polystyrene beads $\left(\sim 2 \times 10^{-10} \mathrm{esu}\right)$ is 2 orders of magnitude smaller than that of gold nanoparticles $\left(\sim 5 \times 10^{-8} \mathrm{esu}\right),{ }^{36}$ a clear separation of the off-axis trapping sites of the polystyrene beads is not resolved. The Kerr effect that modifies the index of refraction and, hence, the direction of the light field, leading to such off-axis ejection, requires higher-level theoretical works; and it will be the subject of further study. Importantly, eqs $1 \mathrm{a}-1 \mathrm{c}$ and 2 suggest the models of the transient optical forces and the impulsive peak power as a function of pulse width. Since average laser power, hence laser power per pulse, is set to be essentially the same, and since the impulsive peak power is inversely related to the pulse width, the magnitudes of all the optical forces are lower with widening the pulse width. Notably, $F_{\text {temp }}$ is further reduced as this force is additionally inversely proportional to the square of the pulse width (eq 1c). Thus, $F_{\text {grad }}$ and $F_{\text {scatt }}$ forces reduce by 15 and $32 \%$, respectively, when the pulse width increases from 85 to 100 and $125 \mathrm{fs}$, and at the same conditions $F_{\text {temp }}$ force reduces by 40 and $70 \%$.

The lower magnitude of $F_{\text {grad }}$ leads to a shallower potential trapping well and, hence, results in fewer numbers of nanoparticles optically trapped in the trapping site (Figure 2). This normal feature is consistent with an abrupt decrease in the scattering light intensity at the focal spot upon widening the pulse width. More importantly, in optical trapping with laser pulses, both the repulsive force, $\left|F_{\text {scatt }}+F_{\text {temp }}\right|$, and ratio of the repulsive to attractive force, $\left|F_{\text {scatt }}+F_{\text {temp }}\right| /\left|F_{\text {grad }}\right|$, depend on the pulse width. As in our numerical approaches, we have shown that $\left|F_{\text {scatt }}\right| \cong\left|F_{\text {temp }}\right| \ll\left|F_{\text {grad }}\right| ;^{30}$ here we can estimate that, upon widening the pulse width from 85 to 100 and $125 \mathrm{fs}$, the $\mid F_{\text {scatt }}+$ $F_{\text {temp }} \mid$ decreases by 27 and $50 \%$ and the $\left|F_{\text {scatt }}+F_{\text {temp }}\right| /\left|F_{\text {grad }}\right|$ ratio decreases by 14 and $27 \%$. Considering that the ejection and directional migration of the nanoparticles outside the focal spot are strongly determined by the magnitude of repulsive force in the front of the attractive forces in the trapping site, the decrease in the $\left|F_{\text {scatt }}+F_{\text {temp }}\right|$ upon widening the pulse width from 85 to 100 and 125 fs can be qualitatively accounted for the shorter migration distance of the ejected nanoparticles by 28 and $68 \%$ (Figure 2). This finding clearly shows that the laser pulses with widened pulse width are much more difficult to eject the nanoparticles out of the trapping site.

It must be pointed out that at the pulse width longer than $125 \mathrm{fs}$, at which the $\left|F_{\text {scatt }}+F_{\text {temp }}\right| /\left|F_{\text {grad }}\right|$ ratio is much lower than that at $85 \mathrm{fs}$, optically trapped nanoparticles is detected but the directional ejections of a discernible amount of nanoparticles out of the trapping site are never observed, similarly to the optical phenomenon with the cw lasers. Here, we should note that since the optical trapping of nanoparticles by the laser pulses is primarily related to the transient $F_{\text {grad }}$ $F_{\text {scatt }}$ and $F_{\text {temp }}$ forces, invoking not only the $\left|F_{\text {scatt }}+F_{\text {temp }}\right| / \mid$ $F_{\text {grad }} l$ ratio but also the impulsive peak power of the laser pulses as well as relaxation and thermal diffusion of the nanoparticles during interval times between consecutive pulses, optical 


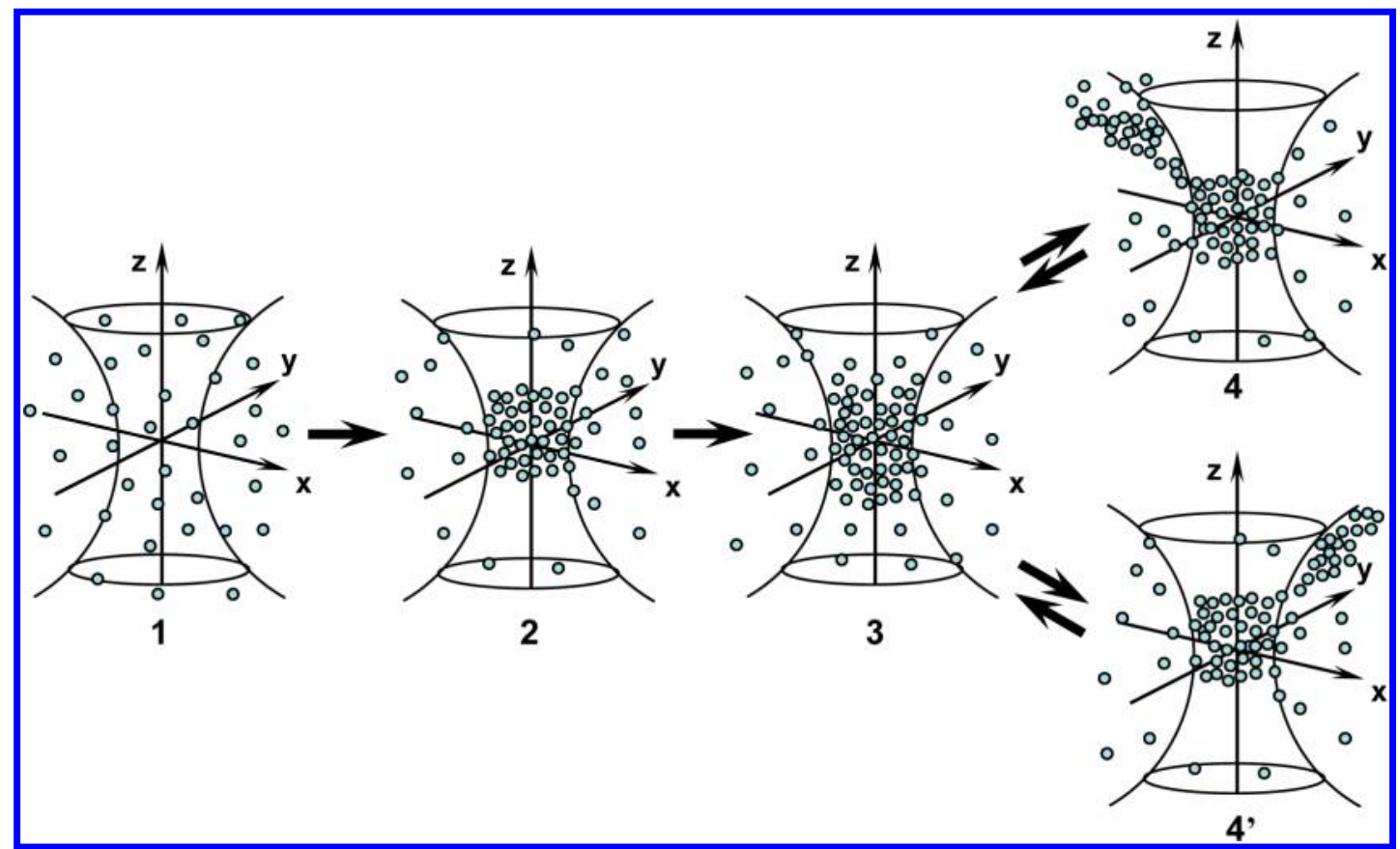

Figure 4. Models of optical confinement and controllable ejection dynamics of polystyrene nanoparticles by tightly focused ultrashort laser pulses with polarization vector along the $x$-axis. A large amount of nanoparticles suspended in water solution (1) are accumulated by the pulse train with high repetition rate and are compacted into a transient assembly in the micrometer-sized trapping site (2). When size of assembly reach a certain level (3), parts of the assembly are ejected out from the trapping site along the $-y(4)$ or $+y$-axis $\left(4^{\prime}\right)$ on the yz-plane, depending on asymmetric shape of the assembly.

trapping dynamics of the nanoparticles by laser pulses with the widened pulse widths should be distinct from that by the conventional cw laser (by which $F_{\text {temp }}$ is naturally neglected). ${ }^{27,30}$ This viewpoint has been demonstrated in optical trapping of $\mathrm{nm}$-sized quantum dots with laser pulses in ps regime. $^{26}$

Theoretical evaluation on the dynamic radiation forces exerted on Rayleigh particles optically trapped by ultrashort laser pulses with pulse width of $0.01-1$ ps has been reported by Wang et al. ${ }^{37}$ In the study, the dynamic radiation forces of $F_{\text {grad }}$ $F_{\text {scatt }}$ and $F_{\text {temp }}$ during the pulse width has been confronted with other forces, including friction, thermal diffusion, and buoyancy forces. The distribution of electric field at the focal spot by a high NA objective, however, has been omitted and evaluation of the dynamic radiation forces has been limited along the beam axis. The important finding is that the pulse-width dependence drastically appears for small-sized (5-nm) Rayleigh particles, while the trapping behavior is independent of the pulse width for large-sized (50-nm) Rayleigh particles. On the contrary, by taking the distribution of electric field at the focal spot, we found that the off-axis repulsive forces overcome the gradient force, and the magnitudes of the optical forces are determined by pulse width. ${ }^{27,30}$ In accordance with our theoretical model, indeed experimental results show the optical trapping and directional ejection of the large-sized (50-nm) Rayleigh particles with clear pulse-width dependences.

4.2. Number of Optically Trapped Nanoparticles Governing Their Ejections. The results presented in Section 3.2 vividly indicate that the optically controlled directional ejection of nanoparticles is also the material-side phenomenon. The key observation is that optically trapped nanoparticles are ejected only when the number of nanoparticles in the trapping site exceeds a certain level (Figure 3C). The existence of a definite threshold may, therefore, reveal how the nanoparticles are trapped and ejected out of the trapping site. Here, we consider several physical parameters in the framework of the particle dynamics, including attractive and repulsive forces, thermal diffusion, and particle assembly, as follows.

The nanoparticles located within the focal spot area interact with a transient electromagnetic field of each laser pulse, and they are attracted to the beam center by the generated gradient force particularly along the polarization vector. The force takes place only during the ultrashort pulse duration, thus they would be displaced in short distances in the close vicinity of the focal spot. Considering the thermal diffusions, in $12.5 \mathrm{~ns}$ interval time between two pulses, translocation of the nanoparticles was estimated to be $\sim 4 \mathrm{~nm}^{2} .^{27}$ Because the translocation is much smaller than cross section of the trapping site, $l_{\mathrm{w}}^{2} \cong 4 \mu \mathrm{m}^{2}$ (where $l_{\mathrm{w}} \cong 5 \times w_{0}, l_{\mathrm{w}}$ is the width of the trapping potential well, and $w_{0}$ is the beam waist), the nanoparticles do not move substantially from the trapping site during the interval time and severe destabilization of the optical trapping due to thermal diffusion can be excluded. This means that the transient gradient force of each laser pulse can attract the nanoparticles to the trapping site, and that the high-repetition-rate pulse train $\left(2.7 \times 10^{6}\right.$ pulses per integrating time of a video frame) accumulates a large number of nanoparticles in $\sim 1.2-\mu \mathrm{m}^{3}$-sized trapping site, which can be filled up roughly by at most $8 \times 10^{3}$ nanoparticles. It is noteworthy that the high particle density in the focal spot can give rise to multiple scattering, but approximation in eqs $1 \mathrm{a}-1 \mathrm{c}$ is limited on the optical forces exerted on a single particle by the incident laser pulses without considering the multiple scattering effects. Because such evanescence fields of nondiffraction limited light can create other optical attractive or repulsive forces, ${ }^{38}$ in addition to the force directly exerted by the incident laser beam (eqs $1 \mathrm{a}-1 \mathrm{c}$ ), the particles may experience smaller optical forces induced by the scattered light from other particles, giving rise additional 
forces to trap or eject the particles. Importantly, the highly confined nanoparticles may optically form a transient assembly. While further evaluations to resolve such assembly formations by the laser pulses-trapping are desirable in the future, we recall that by monitoring the diffusion constant of the optically trapped dye-doped polystyrene nanoparticles under cw laser trapping such assembly formations have been pointed out. ${ }^{14,15}$ Because the detected scattering light outside the trapping site is attributed to assembly of a discernible amount of nanoparticles, the current result indicates that whenever the trapping site is filled up by a large assembly, parts of the assembly are ejected by the repulsive forces and flow into the surrounding. As the transient assembly does not necessarily have symmetric shapes, the parts are ejected only in one direction at one time or in opposite direction at the other time, as illustrated in Figure 4. The balance of the two directional ejections in long observation window indicates the same probability and equal repulsive forces along the two opposing directions perpendicular to the polarization vector, as predicted by the theoretical model. ${ }^{27,30}$

Subsequent filling of the trapping site after an ejection is the key process to trap and eject large numbers of particles continuously. The filling time is strongly governed by the particle density. At high particle density, because of a sufficient supply of nanoparticles to the trapping site, the ejection and subsequent filling take place repetitively within time scales much shorter than the integrating time of the video frame. Thus, the formations of assembly and their directional ejections are almost constant in each video frame. At lower particle densities, as the probability of finding the nanoparticles around the focal spot becomes lower, the supply of nanoparticles to the focal spot is abruptly reduced. Subsequent filling of the trapping site thus includes incubation times, which may invoke movement of a large number of nanoparticles from the region with higher density to the bleached area around the focal spot. As the results, the formation of a large assembly has some interval times and diffusions of nanoparticles by accompanying microfluidic flow around the focal spot are suppressed. This weak microfluidic flow, in turn, causes retrapping of the ejected nanoparticles, as clearly indicated by higher fluctuations in the number of optically trapped nanoparticles.

\section{CONCLUSIONS}

In this research, with image analysis of the Rayleigh scattering, we have experimentally demonstrated the effects of prominent factors governing the confinement and directional ejections of polystyrene nanoparticles in the fs laser trapping. We demonstrated that the pulse width is one of the key parameters for control over the optical confinement and ejections of polystyrene nanoparticles through the adjustment of the magnitudes of transient optical forces as well as the ratio between repulsive and attractive forces. We also found that the directional ejections of the nanoparticles out of the trapping site occurred when the number of confined nanoparticles exceeded a certain level. This observation offers an interpretation that particle density plays a critical role in subsequent filling of the trapping site after the ejection, taking into account of movement of a large number of nanoparticles from the region with higher density to the bleached area around the focal spot. Our future goal is to investigate the fs laser pulses trappinginduced formations of transient assembly that may be controlled by modifying the repetition rate of the laser pulses, the results of which will be published in the near future.

\section{ASSOCIATED CONTENT}

\section{Supporting Information}

Videos of the directional ejections of nanoparticles out of the trapping site for different particle densities (V1: $15.2 \times 10^{2}$ / $\mu \mathrm{m}^{3}$; V2: $3.8 \times 10^{2} / \mu \mathrm{m}^{3}$; and V3: $\left.1.1 \times 10^{2} / \mu \mathrm{m}^{3}\right)$. This material is available free of charge via the Internet at http:// pubs.acs.org.

\section{AUTHOR INFORMATION}

\section{Corresponding Author}

*E-mail: anwar.usman@kaust.edu.sa (A.U.); masuhara@ masuhara.jp (H.M.).

\section{Notes}

The authors declare no competing financial interest.

\section{ACKNOWLEDGMENTS}

The financial supports from the Ministry of Education of Taiwan (MOE-ATU Project; National Chiao Tung University), the National Science Council of Taiwan (Grant No. NSC 1002113-M-009-001), and Foundation of the Advancement for Outstanding Scholarship of Taiwan to H.M. are gratefully acknowledged.

\section{REFERENCES}

(1) Ashkin, A. Acceleration and Trapping of Particle by Radiation Pressure. Phys. Rev. Lett. 1970, 24, 156-159.

(2) Ashkin, A.; Dziedzic, J. M.; Bjorkholm, J. E.; Chu, S. Observation of a Single-Beam Gradient Force Optical Trap for Dielectric Particles. Opt. Lett. 1986, 11, 288-290.

(3) Ashkin, A. History of Optical Trapping and Manipulation of Small-Neutral Particle, Atoms, and Molecules. IEEE J. Quantum Electron. 2000, 6, 841-856.

(4) Molloy, J. E.; Padgett, M. J. Lights, Action: Optical Tweezers. Contemp. Phys. 2002, 43, 241-258.

(5) Grier, D. G. A Revolution in Optical Manipulation. Nature 2003, $424,810-816$.

(6) Neuman, K. C.; Block, S. M. Optical Trapping. Rev. Sci. Instrum. 2004, 75, 2787-2809.

(7) Dholakia, K.; Reece, P.; Gu, M. Optical Micromanipulation. Chem. Soc. Rev. 2008, 37, 42-55.

(8) Sugiyama, T.; Yuyama, K.; Masuhara, H. Laser Trapping Chemistry: From Polymer Assembly to Amino Acid Crystallization. Acc. Chem. Res. 2012, 45, 1946-1954.

(9) Hotta, J.; Sasaki, K.; Masuhara, H. A Single Droplet Formation from Swelled Micelles by Radiation Pressure of a Focused Infrared Laser Beam. J. Am. Chem. Soc. 1996, 118, 11968-11969.

(10) Hofkens, J.; Hotta, J.; Sasaki, K.; Masuhara, H.; Iwai, K. Molecular Assembling by the Radiation Pressure of a Focused Laser Beam: Poly(N-isopropylacrylamide) in Aqueous Solution. Langmuir 1997, 13, 414-419.

(11) Ito, S.; Yoshikawa, H.; Masuhara, H. Laser Manipulation and Fixation of Single Gold Nanoparticles in Solution at Room Temperature. Appl. Phys. Lett. 2002, 80, 482-484.

(12) Urban, A. S.; Lutich, A. A.; Stefani, F. D.; Feldmann, J. Laser Printing Single Gold Nanoparticles. Nano Lett. 2010, 10, 4794-4798.

(13) Bartlett, P.; Henderson, S. Three-Dimensional Force Calibration of a Single-Beam Optical Gradient Trap. J. Phys.: Condens. Matter 2002, 14, 7757-7768.

(14) Hosokawa, C.; Yoshikawa, H.; Masuhara, H. Optical Assembling Dynamics of Individual Polymer Nanospheres Investigated by SingleParticle Fluorescence Detection. Phys. Rev. E 2004, 70, 061410.

(15) Hosokawa, C.; Yoshikawa, H.; Masuhara, H. Cluster Formation of Nanoparticles in an Optical Trap Studied by Fluorescence Correlation Spectroscopy. Phys. Rev. E 2005, 72, 021408. 
(16) Ito, S.; Tanaka, Y.; Yoshikawa, H.; Ishibashi, Y.; Miyasaka, H.; Masuhara, $\mathrm{H}$. Confinement of Photopolymerization and Solidification with Radiation Pressure. J. Am. Chem. Soc. 2011, 113, 14472-14475.

(17) Rungsimanon, T.; Yuyama, K.; Sugiyama, T.; Masuhara, H.; Tohnai, N.; Miyata, M. Control of Crystal Polymorph of Glycine by Photon Pressure of a Focused Continuous Wave Near-Infrared Laser Beam. J. Phys. Chem. Lett. 2010, 1, 599-603.

(18) Tsuboi, Y.; Shoji, T.; Kitamura, N. Optical Trapping of Amino Acids in Aqueous Solutions. J. Phys. Chem. C 2010, 114, 5589-5593.

(19) Masuhara, H.; Sugiyama, T.; Rungsimanon, T.; Yuyama, K.; Miura, A.; Tu, J.-R. Laser-Trapping Assembling Dynamics of Molecules and Proteins at Surface and Interface. Pure Appl. Chem. 2011, 83, 869-883.

(20) Pang, Y.; Gordon, R. Optical Trapping of $12 \mathrm{~nm}$ Dielectric Spheres Using Double-Nanoholes in a Gold Film. Nano Lett. 2012, 12, 402-406.

(21) Garetz, B. A.; Aber, J. E.; Goddard, N. L.; Young, R. G.; Myerson, A. S. Non-photochemical, Polarization-Dependent, LaserInduced Nucleation in Supersaturated Aqueous Urea Solutions. Phys. Rev. Lett. 1996, 77, 3475-3476.

(22) Garetz, B. A.; Matic, J.; Myerson, A. S. Polarization Switching of Crystal Structure in the Nonphotochemical Light-Induced Nucleation of Supersaturated Aqueous Glycine Solutions. Phys. Rev. Lett. 2002, 89, 175501.

(23) Matic, J.; Sun, X.; Garetz, B. A.; Myerson, A. S. Intensity, Wavelength, and Polarization Dependence of Nonphotochemical Laser-Induced Nucleation in Saturated Aqueous Urea Solutions. Cryst. Growth Des. 2005, 5, 1565-1567.

(24) Adachi, H.; Takano, K.; Hosokawa, Y.; Inoue, T.; Mori, Y.; Matsumura, H.; Yoshimura, M.; Tsunaka, Y.; Morikawa, M.; Kanaya, S.; et al. Laser Irradiated Growth of Protein Crystal. Jpn. J. Appl. Phys. 2003, 42, L798-L800.

(25) Agate, B.; Brown, C. T. A.; Sibbett, W.; Dholakia, K. Femtosecond Optical Tweezers for In-Situ Control of Two-Photon Fluorescence. Opt. Express 2004, 12, 3011-3017.

(26) Pan, L.; Ishikawa, A.; Tamai, N. Detection of Optical Trapping of CdTe Quantum Dots by Two-Photon-Induced Luminescence. Phys. Rev. B 2007, 75, 161305.

(27) Usman, A.; Chiang, W.-Y.; Masuhara, H. Optical Trapping and Polarization-Controlled Scattering of Dielectric Spherical Nanoparticles by Femtosecond Laser Pulses. J. Photochem. Photobiol. A: Chem. 2012, 234, 83-90.

(28) Usman, A.; Chiang, W.-Y.; Masuhara, H. Femtosecond Trapping Efficiency Enhanced for Nanosized Silica Spheres. Proc. SPIE 2012, 8458, 845833.

(29) Jiang, Y.; Narushima, T.; Okamoto, H. Nonlinear Optical Effects in Trapping Nanoparticles with Femtosecond Pulses. Nat. Phys. 2010, 6, 1005-1009.

(30) Usman, A.; Chiang, W.-Y.; Masuhara, H. Optical Trapping of Nanoparticles with Ultrashort Laser Pulses. Sci. Prog. 2013, 96, 1-18.

(31) Seol, Y.; Carpenter, A. E.; Perkins, T. T. Gold Nanoparticles: Enhanced Optical Trapping and Sensitivity Coupled with Significant Heating. Opt. Lett. 2006, 31, 2429-2431.

(32) Kyrsting, A.; Bendix, P. M.; Oddershede, L. B. Mapping 3D Focal Intensity Exposes the Stable Trapping Positions of Single Nanoparticles. Nano Lett. 2013, 13, 31-35.

(33) Miles, R. E. H.; Rudic, S.; Orr-Ewing, A. J.; Reid, J. P. Influence of Uncertainities in the Diameter and Refractive Index of Calibration Polystyrene Beads on the Retrieval of Aerosol Optical Properties using Cavity Ring Down Spectroscopy. J. Phys. Chem. A 2010, 114, 70777084.

(34) Davis, L. W. Theory of Electromagnetic Beams. Phys. Rev. A 1979, 19, 1177-1179.

(35) Novotny, L.; Hecht, B. Principles of Nano-Optics; Cambridge Univ. Press: Cambridge, U.K., 2006.

(36) Bloemer, M. J.; Haus, J. W.; Ashley, P. R. Degenerate of FourWave Mixing in Colloidal Gold as a Function of Particle Size. J. Opt. Soc. Am. B 1990, 7, 790-795.
(37) Wang, L.-G.; Chai, H.-S. Revisit on Dynamic Radiation Forces Induced by Pulsed Gaussian Beams. Opt. Express 2011, 19, 1438914402.

(38) Jaquay, E.; Martinez, L. J.; Mejia, C. A.; Povinelli, M. L. LightAssisted, Templated Self-Assembly Using a Photonic-Crystal Slab. Nano Lett. 2013, 13, 2290-2294. 